\title{
Adaptive image deblurring with ringing control
}

\author{
Andrey S. Krylov, Andrey V. Nasonov \\ Laboratory of Mathematical Methods of Image Processing \\ Faculty of Computational Mathematics and Cybernetics, Moscow Lomonosov State University \\ krylecs.msu.ru; nasonovecs.msu.ru
}

\begin{abstract}
The paper presents an adaptive image deblurring method with ringing control. Images are split in analogy with unsharp mask into low- and high-frequency components. Edges are sharpened in low-frequency domain using deconvolution with Total Variation constraint. Highfrequency information is amplified using ringing level control.
\end{abstract}

\section{Introduction}

The problem of image deblurring is to restore highfrequency information from blurred image. It is posed mathematically as reconstruction of the initial image $z$ from blurry and noisy observation $u$ using the model

$$
u=H z+n,
$$

where $H z=H * z$ is a convolution with a blur operator, $n$ is a noise. In blind deconvolution problem, $H$ is unknown and has to be estimated.

The problem of image deblurring is often posed as an inverse problem to (1). This problem is ill-posed, and regularization method $[1,10]$ is used to solve it:

$$
z_{\text {reg }}=\arg \min _{z}\left(\|H z-u\|_{2}^{2}+\alpha \Omega[z]\right),
$$

where $\Omega[z]$ is a stabilizer which puts imposes additional constraints to the solution, $\alpha$ is a regularization parameter. The simplest choice of the stabilizer is the Total Variational functional $T V[z]=\int|\nabla z| \partial z$. It was first used for the problem of image enhancement by Rudin, Osher and Fatemi [9].

One of the main problems of image regularization methods is the choice of regularization parameter $\alpha$. Low values result in ringing and noise amplification while high values result in high frequency details loss [2].

Linear unsharp mask $z_{M}=H u+q(u-H u)$ is used in non-iterative deblurring for Gauss blur model [5]. It amplifies the high-frequency information $(u-H u)$ by factor $q$. Unsharp mask has many advantages. It is a linear spaceinvariant filter, which is computationally inexpensive and can be easily implemented as a spatial-domain convolution. The main disadvantage of this method is strong noise amplification and ringing effect.

We present an image deblurring algorithm which combines both regularization method and unsharp mask and uses ringing level control to suppress ringing effect. The key idea of the proposed method is to split the image into low-frequency image and high-frequency image, apply regularization-based sharpening to low-frequency image and combine the sharpened low-frequency image with amplified high-frequency image.

\section{The Proposed Algorithm}

We consider the problem of image deblurring for uniform Gaussian blur $H=G$, where the Gauss radius $\sigma$ is unknown and has to be estimated.

The proposed algorithm consists of the following steps:

1. Estimate Gauss radius $\sigma$ of the blur operator $G$. This is performed using the analysis of the edge width (see Section 3).

2. Split the given image $u$ into low-frequency $u_{L}$ and high-frequency $u_{H}$ components. Low-frequency image $u_{L}$ is constructed by applying the blur operator $G$ to the given image $u: u_{L}=G u$. High-frequency image is calculated as the difference $u_{H}=u-H u$.

3. Apply regularization-based sharpening $S$ to the lowfrequency image $z_{L}=S u_{L}$ (see Section 4).

4. Amplify high-frequency information by a factor $q$ and add it to the sharpened low-frequency image $z_{R}=S u_{L}+$ $q u_{H}$. Parameter $q$ is chosen in accordance with ringing level function (see Section 5).

\section{Gauss radius estimation}

\subsection{Edge width analysis}

The term 'edge width' does not have a certain definition. The simplest approach used in [4] is to find local minimum 
and local maximum near the edge center. This approach does not provide stable results for blurred and noisy edges. In [11], the edge is modeled by a special function, but it does not fit our needs.

For one-dimensional edge

$$
\bar{f}(x)= \begin{cases}f_{0}, & x \leq x_{0}, \\ f_{0}+\frac{\left(f_{1}-f_{0}\right)\left(x-x_{0}\right)}{x_{1}-x_{0}}, & x_{0}<x<x_{1} \\ f_{1}, & x \geq x_{1}\end{cases}
$$

we define edge width as $w(\bar{f})=x_{1}-x_{0}$.

To define edge width $w$ for an arbitrary edge $f(x)$, we approximate it by the edge $\bar{f}(x)(2)$. We seek for minimum $f_{0}$ and maximum values $f_{1}$ of $f(x)$ in a neighborhood of the edge center, reducing for simplicity the edge to the case $f_{0}=0, f_{1}=1$. The size of this neighborhood is chosen a priori and represents the maximum considered edge width.

Next we seek for coordinates of intersections of $y=$ $f(x)$ with $y_{0}=1 / 4$ and with $y_{1}=3 / 4$, draw a line through these points and find $x_{0}$ and $x_{1}$ as it is shown in Fig. 1. In the case of multiple intersections we take the average of intersection points. We consider the obtained value as the edge width estimation.

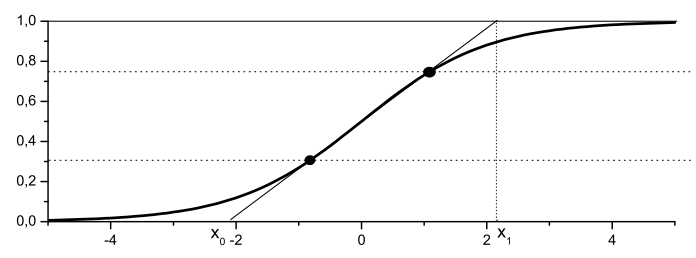

Figure 1. Edge width estimation. For the shown case, the estimated edge width $w$ is 4.3 pixels.

\subsection{The dependence of edge width on Gauss radius of the blur operator}

To estimate Gauss radius $\sigma$ of the blur operator $G$, we use edge width analysis. We performed an experiment to find the dependence of edge width $w$ on Gauss radius $\sigma$. Gauss blur operators with different $\sigma$ were applied to the images from the test image set and the average edge width $w$ was calculated for every $\sigma$. The results are shown in Fig.2.

The function $w(\sigma)$ is close to the linear function for $\sigma \geq 3$. So we approximate the function $w(\sigma)$ by the linear function $w(\sigma)=\frac{1}{k} \sigma$ and empirically calculate the coefficient $k$. For the test image set, the value $k$ is 0.45 . We used the test image set consisted of standard images 'House', 'Lena', 'Boat', 'Peppers' and 'Barbara'.

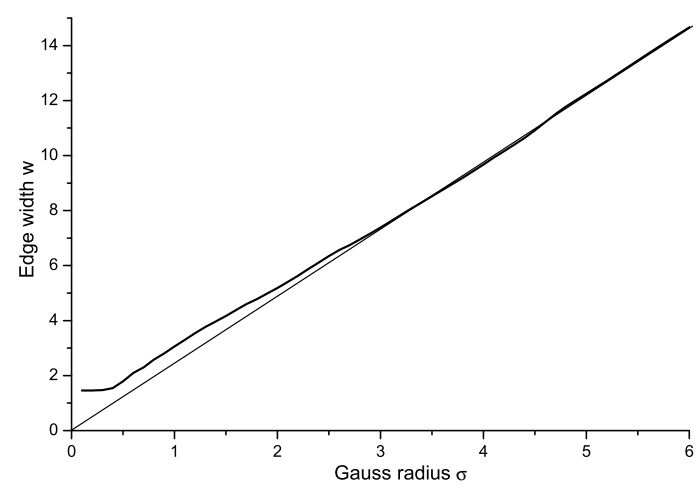

Figure 2. The dependence of edge width $w$ on Gauss filter radius $\sigma$ for the test image set.

\subsection{Gauss radius estimation for $2 \mathrm{D}$ images} radius

Using the equation $w(\sigma)=\frac{1}{k} \sigma$, we can estimate the

$$
\sigma(w)=k w
$$

for a single edge with edge width $w$. But in 2D case, there are lots of edges, and the estimated edge width is different for different edges. It is caused, for example, by noise, nonuniform blur or different edge sharpness.

In this paper, we consider the problem of Gauss radius estimation for the case of uniform blur. To analyze the edge width, only sharp and isolated edges are used to reduce the influence of nearby edges and noise.

We use the following algorithm:

1. First, we perform edge detection and extract edge normal cross-sections $f_{i}, i=1,2, \ldots, N$ for strong isolated edges. We use the algorithm from [7].

2. Next we estimate edge widths $w_{i}=w\left(f_{i}\right)$ for all cross-sections and calculate $\sigma_{i}=k w_{i}$.

3. Then estimate $\sigma$ as the value of the highest density of $\left\{\sigma_{i}\right\}$ distribution.

\section{Regularization-based Sharpening}

TV regularization shows good results for the problem of image deblurring. The first advantage is that it does not smooth the edges. The resulting image tends to piece-wise flat solution in the case of increasing regularization parameter. The second advantage is that TV is closely related with ringing artifact [6]. It makes possible to control ringing artifact by regularization parameter.

\subsection{Total Variation}

In one-dimensional case, Total Variation functional is defined as

$$
T V(f)=\int_{-\infty}^{\infty}\left|f^{\prime}(x)\right| d x .
$$


In the discrete case $-\infty<\ldots<x_{k-1}<x_{k}<\ldots<$ $\infty$, it looks as:

$$
T V(f)=\sum_{k=-\infty}^{\infty}\left|f\left(x_{k}\right)-f\left(x_{k-1}\right)\right| .
$$

For two-dimensional image, we use the following representation:

$$
T V(z)=\sum_{i, j}\left|z_{i+1, j}-z_{i, j}\right|+\sum_{i, j}\left|z_{i, j+1}-z_{i, j}\right| .
$$

\subsection{TV Deblurring}

If we consider the step edge (2) and its blurred version, it is obvious that TV of the edge does not depend on the radius of Gaussian blur and is equal to $\left|f_{1}-f_{0}\right|$. Thus, we assume, that deblurring of a blurred step edge does not change its $T V$.

Real images contain not only step edges but also fine details, so TV deblurring cannot be applied to the problem of image deblurring directly. Nevertheless we consider the low-frequency component $u_{L}$ of the given image as an image which consists only of step edges so we apply TV deblurring to it:

$$
z_{L}=\arg \min _{T V(z) \leq T V\left(u_{L}\right)}\left\|G(G z)-u_{L}\right\|_{2}^{2},
$$

where $G(G z)$ and $u_{L}$ are calculated using our $\sigma(w)$ estimation.

The example of low-frequency edge sharpening is given in Fig.3.

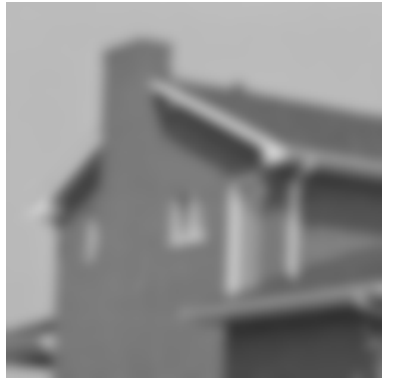

Low-frequency information

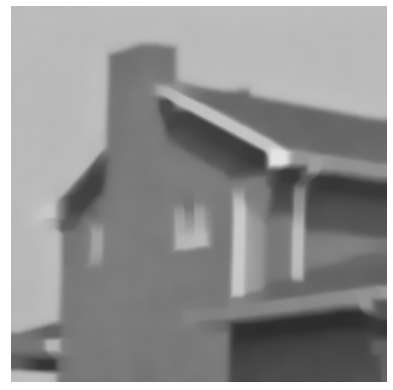

TV deblur result
Figure 3. TV deblur of the low-frequency image component

\section{High-frequency Amplification}

The high-frequency information $u_{H}$ is amplified with a factor of $q$ and then added to the result of sharpened lowfrequency image $z_{L}$. The main problem is that high $q$ results in noise amplification and ringing artifact. We consider only the problem of ringing artifact here.
The main idea is to calculate image ringing level. Ringing level is estimated using scale-space TV analysis of strong isolated edges [7, 8].

Parameter $q$ is increased until this value reaches the ringing threshold of the ringing level function.

\section{Results}

The results of the proposed algorithm are illustrated with the image 'house' from the test image set and with the image 'fish'. To estimate the quality of deblurring method, we analyze the quality in edge areas using special metrics from [7].

$B E P$ (Basic Edges Points RMSE) value calculates the square root of the average square error in the edge points area. $B E N$ (Basic Edges Neighborhood RMSE) value calculates the error in edge neighborhood. It is the area where ringing effect usually appears. These metrics are illustrated in Fig.4. White area is the area of the $B E P$ calculation, grey area is the area of the $B E N$ calculation, dark grey lines show the edges.

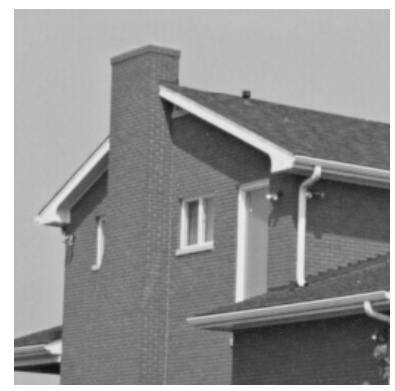

Reference image

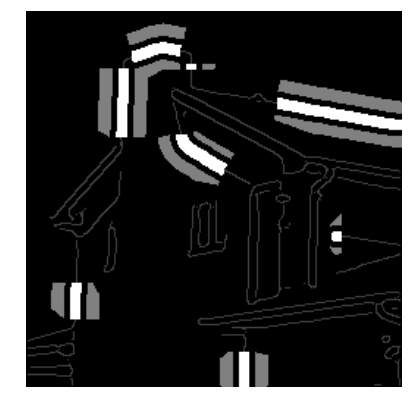

$\mathrm{BEP}$ (white) and BEN (gray) areas
Figure 4. $B E P$ and $B E N$ metrics illustration.

The original images were blurred using Gauss filter with radius $\sigma=3$. The proposed method and unsharp mask were applied to these images. The results are illustrated in Fig.5. The proposed deblurring method shows better $B E P$ than unsharp mask, but it slightly increases the $B E N$ level. 

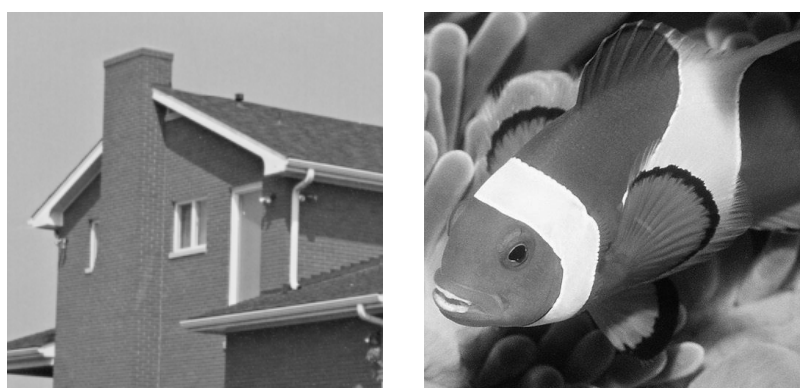

Reference images
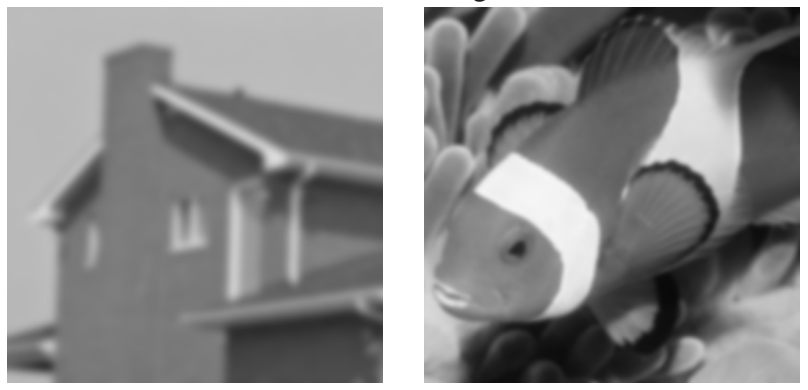

$\mathrm{BEP}=18.38, \mathrm{BEN}=4.16$

$\mathrm{BEP}=24.50, \mathrm{BEN}=6.89$

Blurred images

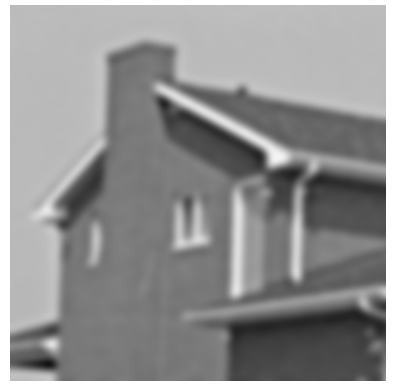

$\mathrm{BEP}=13.42, \mathrm{BEN}=4.15$

Unshar

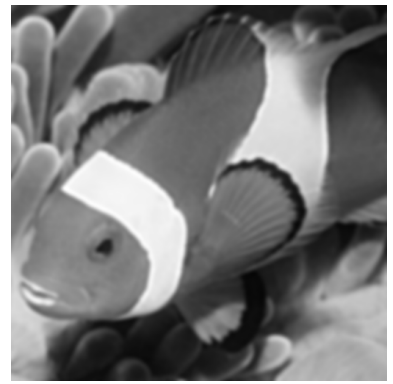

$\mathrm{BEP}=18.70, \mathrm{BEN}=6.43$

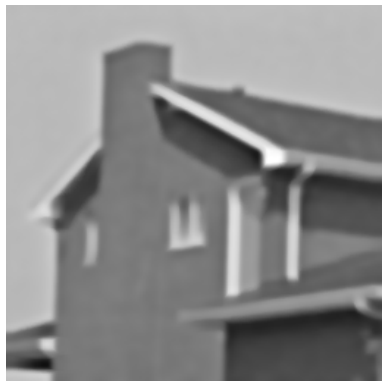

$\mathrm{BEP}=10.28, \mathrm{BEN}=4.68$

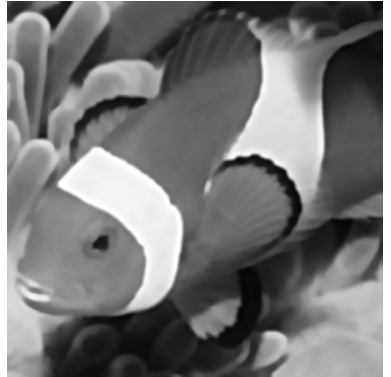

$\mathrm{BEP}=14.07, \mathrm{BEN}=6.45$

Results of the proposed method
Figure 5. Application of the proposed method to the problem of image deblurring.

It can be seen that the proposed deblurring method shows good results in edge areas while unsharp mask works better in non-edge areas. To get the best results, we combine the results of the proposed method $z_{R}$ and of unsharp mask $z_{M}$ :

$$
z_{C}(P)=(1-\alpha(P)) z_{R}(P)+\alpha(P) z_{M}(P),
$$

where $\alpha(P)$ is a function of distance $\rho(P)$ from the point $P$ to the nearest edge:

$$
\alpha(P)= \begin{cases}0, & \rho(P) \leq w / 4, \\ 4 \rho(P) / w-1, & w / 4<\rho(P)<w / 2, \\ 1, & \rho(P) \geq w / 2,\end{cases}
$$

where $w$ is the estimated average edge width.

The results of the combined method are shown in Fig.6. Here we used Canny edge detector [3] with radius 1.

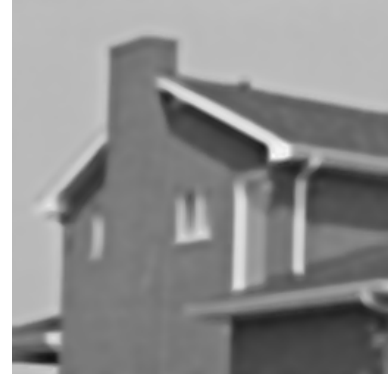

$\mathrm{BEP}=10.05, \mathrm{BEN}=4.15$

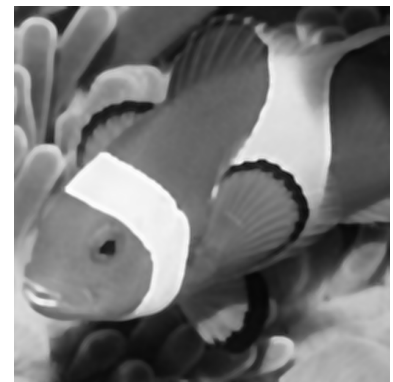

$\mathrm{BEP}=13.38, \mathrm{BEN}=6.44$
Figure 6. Application of the proposed combined method to the problem of image deblurring.

\section{Conclusion}

The suggested adaptive image deblurring method with ringing control shows promising results comparing with unsharp mask method. The future work will include an adjustable procedure of low-frequency domain edges sharpening. High-frequency information amplification using ringing level control also needs additional investigations.

The work was supported by federal target program "Scientific and scientific-pedagogical personnel of innovative Russia in 2009-2013" and RFBR grant 09-01-92470MHKC.

\section{References}

[1] M. Almeida and L. Almeida. Blind deblurring of natural images. IEEE Int. Conf. on Acoustics, Speech and Signal Processing, 2008, pages 1261-1264, 2008. 1

[2] M. Banham and A. Katsaggelos. Digital image restoration. IEEE Signal Proc. Magazine, 14:24-41, 1997. 1

[3] J. Canny. A computational approach to edge detection. IEEE Trans. PAMI, 8:679-714, 1986. 4

[4] M. Khambete and M. Joshi. Blur and ringing artifact measurement in image compression using wavelet transform. Proceedings of World Academy of Science, Engineering and Technology, 20:183-186, 2007. 1 
[5] J. S. Lim. Two-Dimensional Signal and Image Processing, pages 459-463. Prentice-Hall, Englewood Cliffs, NJ, 1990. 1

[6] S. Mallat. A Wavelet Tour of Signal Processing. Academic Press, 1999. 2

[7] A. Nasonov and A. Krylov. Adaptive image deringing. To appear in Graphicon'2009, Moscow, October 2009. 2, 3

[8] A. Nasonov and A. Krylov. Scale space methods of image ringing estimation. To appear in ICIP'09, Cairo, 2009. 3

[9] L. Rudin, S. Osher, and E. Fatemi. Nonlinear total variation based noise removal algorithms. Physica $D$, 60:259-268, 1992. 1

[10] A. N. Tikhonov and V. Y. Arsenin. Solutions of Ill Posed Problems. WH Winston, Washington DC, 1977. 1

[11] P. Zeng and T. Hirata. Distance map based enhancement for interpolated image. Lecture Notes in Computer Science, 2616:429-449, 2003. 2 